\title{
Assessing cardiac preload by the Initial Systolic Time Interval obtained from impedance cardiography
}

\author{
Jan H. Meijer ${ }^{1,3}$, Annemieke Smorenberg ${ }^{2}$, Erik J. Lust ${ }^{2}$, Rudolf M. Verdaasdonk ${ }^{1}$ and A. B. Johan Groeneveld ${ }^{2}$ \\ 1. Department of Physics and Medical Technology, VU University Medical Center, Amsterdam, the Netherlands \\ 2. Department of Intensive Care for Adults, VU University Medical Center, Amsterdam, the Netherlands \\ 3. E-mail any correspondence to: jh.meijer@vumc.nl
}

\begin{abstract}
The Initial Systolic Time Interval (ISTI), obtained from the electrocardiogram (ECG) and impedance cardiogram (ICG), is considered to be a measure for the time delay between the electrical and mechanical activity of the heart and reflects an early active period of the cardiac cycle. The clinical relevance of this time interval is subject of study. This paper introduces a method using ISTI to evaluate and predict the circulatory response to fluid administration in patients after coronary artery bypass graft surgery and presents preliminary results of a pilot study by comparing ISTI with cardiac output $(\mathrm{CO})$ responsiveness. Also the use of the pulse transit time (PTT), earlier recommended for this purpose, was investigated. The results showed an inverse relationship between ISTI and $\mathrm{CO}$ at all moments of fluid administration and also an inverse relationship between the changes $\Delta \mathrm{ISTI}$ and $\Delta \mathrm{CO}$ before and after full fluid administration. No relationships between PTT and $\mathrm{CO}$ or $\Delta \mathrm{PTT}$ and $\Delta \mathrm{CO}$ were found. It is concluded that ISTI is dependent upon preload, and that ISTI has the potential to be used as a clinical parameter assessing preload.
\end{abstract}

Keywords: Bioimpedance, impedance cardiography, initial systolic time interval, cardiac preload, coronary artery bypass graft surgery

\section{Introduction}

\section{Impedance Cardiography}

When the electrical impedance of the thorax is measured, a variation synchronous with the heart activity is observed. The time derivative of this variation of the thoracic impedance is called the Impedance CardioGram (ICG). In extended studies during the last decades numerous researchers have tried to translate the amplitude of the ICGsignal into cardiac stroke volume. Meanwhile, it has become evident that the origin of the signal is too complicated to make an interpretation of the signal variations according to very simplified physiological models such as a volumetric changes of the aorta [1]. However, an interesting aspect of the ICG-signal can be found in the time-relationships, when the moments of occurrence of specific waves in the ECG are compared to specific points in the Electro-CardioGram (ECG). Regardless of the multiple sources of the signal, the ICG reflects the mechanical/hydrodynamical aspects of the cardiac cycle [2], while the ECG reflects the electrical aspect of the heart. Therefore, the time difference between specific moments in the two signals can be interpreted as the time difference between the electrical and mechanical activity of the heart and may contain valuable clues about the functioning of the heart, also as controlled by the autonomic nervous system, and the condition of the circulation. Both ECG and ICG signals can be obtained fast and easily. The measurements are non-invasive, form no burden to the patient and are not restricted to a hospital environment. The time difference can be evaluated under various circumstances, for instance during physical activity, and in various diseases and physiological conditions. The necessary equipment is relatively inexpensive and can be operated without extensive education and training.

\section{Initial Systolic Time Interval}

Several earlier studies in the field of bio-impedance investigated a parameter equivalent to the Pre-Ejection Period (PEP) and obtained from the ICG (ICG-PEP) [3, 4, 5]. The PEP is considered to be a measure for the time delay between the electrical and mechanical activation of the heart. The ICG-PEP is defined as the time difference between the Q-point in the ECG and the B-point in the ICG signal. Pharmacological studies showed that ICG-PEP shortened as sympathetic activity increased, for example when a beta-adrenergic agonist like norepinephrine was administered. In concordance, ICG-PEP increased when a sympathetic antagonist (e.g. metoprolol) was used. However, the marker points used to measure the ICG-PEP are not always present in the signals and may be obscured by noise, therefore making them difficult to detect in an automated way. In order to avoid these problems, another time interval has been introduced: the Initial Systolic Time Interval (ISTI), based on distinct and detectable marker points in both signals [6]. From a theoretical point of view the ISTI depends on three factors: the preload of the heart by way of the Frank-Starling mechanism, the autonomic nervous control, and the afterload of the heart caused by the peripheral resistance. However, the use of the ISTI in many situations has not yet been established. There is a need for observational research to reveal the behaviour of the ISTI in different conditions and populations of patients. 
Cardiac responsiveness to intravenous fluid administration

Intravenous fluid administration is a cornerstone in the treatment of hypotension after cardiac surgery. However, the accuracy of indices used to assess preload and to predict fluid responsiveness has been an issue of discussion [7]. There is a need for parameters that are able to assess preload and to predict fluid responsiveness in patients in the intensive care unit in order to avoid adverse events like pulmonary oedema. Previous studies have reported that a parameter, equivalent to the pulse transit time (PTT), which is obtained from the ECG and the invasively measured arterial pressure $\mathrm{P}_{\mathrm{a}}$, is dependent on the cardiac preload and can be used for this assessment $[8,9]$. The pulse transit time (PTT) as defined in other studies [11] consists of two components: the PEP, which corresponds to the timing from the onset of ventricular depolarisation to the onset of ventricular ejection, and the ventricular transit time (VTT) which defines the period for the arterial pulse wave to travel from the aortic valve to the peripheral arteries. The PTT was reported to reflect variation in PEP originating from changes in thoracic blood volume in head-tilt experiments [11]. The definition PTT $=$ PEP + VTT from this report is used in the present study. The ISTI, obtained from impedance cardiography, is thought to depend on the cardiac preload as well as the PEP [10]. The present study investigates whether ISTI can be used to evaluate and predict responsiveness of the cardiac output to fluid administration and to compare ISTI with the use of PTT. This paper presents the method to use ISTI for this purpose and reports on preliminary results obtained from patients admitted to the intensive care unit after coronary artery bypass surgery [12].

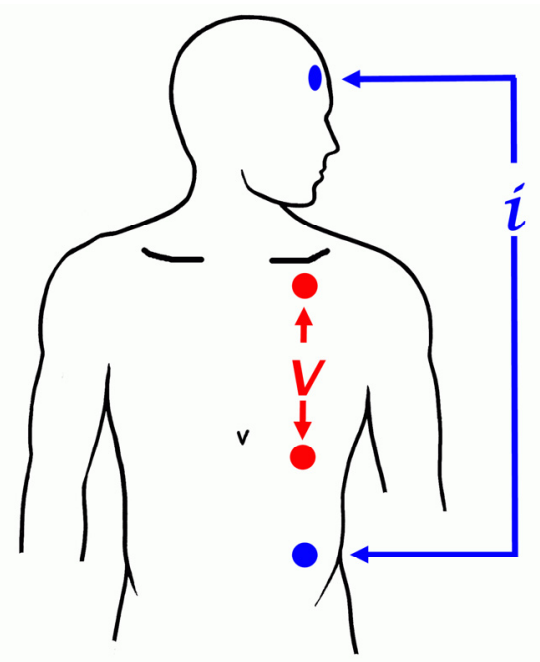

Fig.1: Electrode configuration. A small alternating electrical current of $0.3 \mathrm{~mA}$ r.m.s. and $64 \mathrm{kHz}$ was applied to the thorax by means of the two outer electrodes. The two inner electrodes measured the subsequent electrical voltage difference over the heart. From the time course of this voltage signal the impedance signal was obtained.

\section{Materials and methods}

\section{ICG and ECG signal registration}

The equipment used to record and process ICG- and ECGsignals was designed and constructed at the department of Physics and Medical Technology of the VU University Medical Center in Amsterdam. ICG recordings were made using a tetrapolar electrode system on the left side of the body (figure 1). A very small electrical current $(0.3 \mathrm{~mA}$ r.m.s., $64 \mathrm{kHz}$ ) was applied through the thorax by means of the two outer electrodes. The inner two electrodes were placed immediately below the left clavicula and vertically down at the horizontal level of the processus xiphoideus. These electrodes continuously measured the subsequent electrical voltage difference over the heart. From this voltage difference the impedance signal and ICG were computed on-line. The two outer electrodes were placed at the forehead and at least $20 \mathrm{~cm}$ vertically below the lower measuring electrode at the level of the anterior superior iliac spine. This placement ensured a large distance between the current applying outer electrodes and voltage measuring inner electrodes. The region of measurement must be clear of inhomogeneities in the electrical field that are present in the body in the vicinity of current applying electrodes. This requires an adequate distance between the measuring and current applying electrodes [13]. The ECG signal, also required for the determination of ISTI, was derived simultaneously from the two inner electrodes. The measurement of ISTI has been described earlier by Meijer et al. [6].

A typical example of a simultaneous recording of an ICG and an ECG is shown in figure 2. The ICG-signal is the first time-derivative of the impedance variations across the heart. The figure shows the R-point in the ECG and the C-point in the ICG. The Initial Systolic Time Interval (ISTI) is defined as the period of time between those R- and C-points. The PTT was obtained by simultaneous ECG recording and arterial pressure wave tracing $\left(\mathrm{P}_{\mathrm{a}}\right)$ obtained from the radial artery, in concordance with Bendjelid et al [8] and Feissel et al. [9]. Both signals are commonly monitored in critically ill patients. The effect of volume expansion was established by measuring cardiac output (CO) by means of a thermodilution technique using a catheter in the arteria pulmonalis. The average of three subsequent CO-measurements was used.

\section{Patients and procedures}

16 clinically hypovolaemic patients, who were admitted to the Intensive Care Unit after coronary artery bypass surgery, were measured during intravenous administration of $2 \times 250 \mathrm{ml}$ of a Gelofusine ${ }^{\circledR}$ solution. The patients were considered to be hypovolaemic if either: a) the systolic bloodpressure was less than $110 \mathrm{mmHg}$; b) the central venous pressure (CVP) was less than $10 \mathrm{mmHg}$; c) the 
pulmonary capillary wedge pressure (PCWP) was less than $12 \mathrm{mmHg}$; d) central or mixed venous $\mathrm{SO}_{2}$ was less than $70 \%$; or e) if isotropic or vasopressor drugs were being used. The measurements of CO, ISTI and PTT were performed at three moments: before infusion, after the first and after the second infusion of $250 \mathrm{ml}$ each. A total change in $\mathrm{CO}$ of more than $5 \%$ was considered as a positive response to fluid administration. A total change in $\mathrm{CO}$ of less than 5\% was considered as a non-response. All patients were intubated and mechanically ventilated. The group consisted of 14 males and 2 females, having an age (mean \pm s.d.) of $68 \pm 11$ years (range 44-84 yrs), height $174 \pm 10 \mathrm{~cm}$ (range 151-188 cm) and weight $84 \pm 15 \mathrm{~kg}$ (range $54-125 \mathrm{~kg}$ ). Exclusion criteria were: abnormal cardiac rhythm or presence of an artificial pacemaker, relevant alterations in medication, and any medical, ethical or practical drawbacks or objections to perform the measurements. The study was approved by the Ethics Committee of the VU University Medical Center Amsterdam. All subjects gave informed consent prior to surgery to participate in the test.

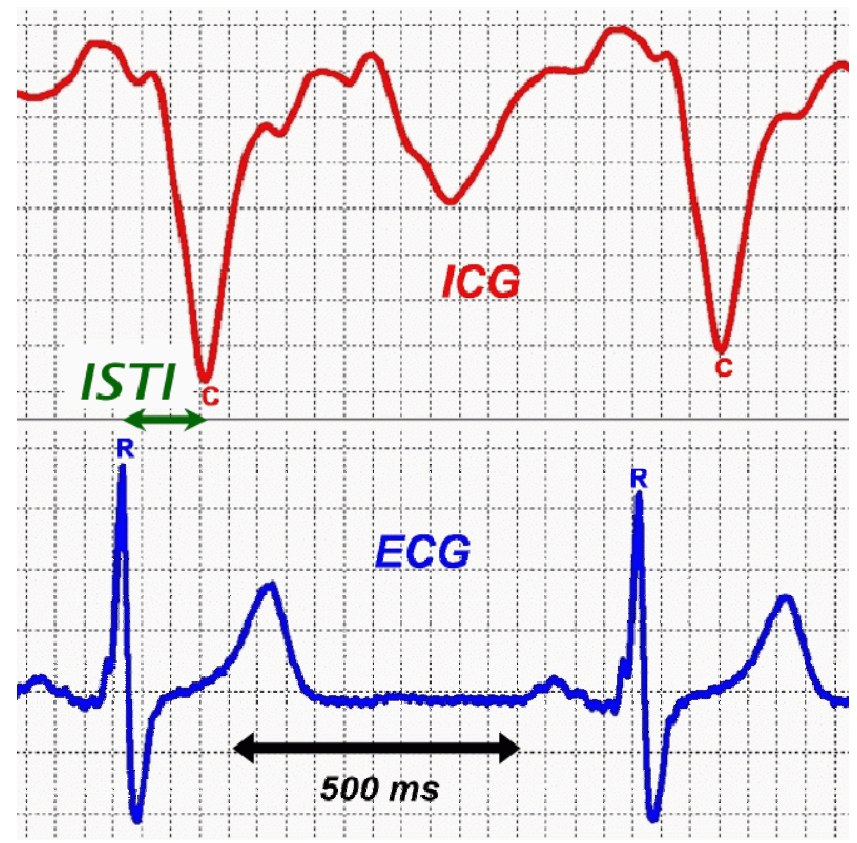

Fig.2: A typical example of simultaneous registration of an Impedance CardioGram (ICG) and an ElectroCardioGram (ECG) (arbitrary units). The marker points R in the ECG and C in the ICG are indicated. From the time difference between these points the Initial Systolic Time Interval (ISTI) is determined, which can be considered as a measure of the time lag between the electrical and mechanical activity of the heart.

\section{Results}

Cardiac output increased in 7 patients, remained unchanged (total change in $\mathrm{CO}$ less than 5\%) in 6 patients and decreased in 3 patients after the full administration of fluid. The mean value of change in cardiac output between moments 3 and 1 was: $\Delta \mathrm{CO}_{31}=0.2 \pm 0.5 \mathrm{l} / \mathrm{min}$. The mean values $( \pm$ S.D.) of the three parameters at the three moments, before infusion (moment 1), after administration of $250 \mathrm{ml}$ (moment 2) and after administration of $500 \mathrm{ml}$ of fluid (moment 3 ), are listed in table 1.

Table 1. Mean values ( \pm S.D.) of the Cardiac Output (CO), Pulse Transit Time (PTT) and Initial Systolic Time Interval (ISTI) at three moments, before and after intravenous administration of a Gelofusine ${ }^{\circledR}$ solution in two steps of $250 \mathrm{ml}$ each.

\begin{tabular}{|l|l|l|l|}
\hline \multicolumn{1}{|c|}{ moment } & CO (L/min) & \multicolumn{1}{c|}{ PTT $(\mathrm{ms})$} & \multicolumn{1}{c|}{ ISTI $(\mathrm{ms})$} \\
\hline before infusion & $4.7 \pm 1.2$ & $190 \pm 9$ & $148 \pm 30$ \\
\hline after $250 \mathrm{ml}$ & $4.9 \pm 1.3$ & $184 \pm 10$ & $151 \pm 28$ \\
\hline after $500 \mathrm{ml}$ & $4.9 \pm 1.3$ & $179 \pm 9$ & $151 \pm 26$ \\
\hline
\end{tabular}

A significant relationship between ISTI and $\mathrm{CO}$ was observed at each of the three moments. These relationships, obtained from least-squares linear regression analysis, are presented in table 2. The observed inverse relationships at the three different moments were found to be consistent. Therefore, the relationship for the pooled data is given in the last row of table 2 and shown in figure 3 . No significant relationships between $\mathrm{CO}$ and PTT and between the changes $\Delta \mathrm{CO}$ and $\triangle \mathrm{PTT}$ were found at the three moments.

Table 2. Relationships between the Initial Systolic Time Interval ISTI (ms) and the Cardiac Output $\mathrm{CO}(\mathrm{L} / \mathrm{min})$ at the three moments before, after the first and after the second intravenous fluid administration of $250 \mathrm{ml}(\mathrm{N}=16)$. The coefficients of correlation $r$ and the levels of significance $p$ are also given. The last row gives the relationship for the pooled data $(\mathrm{N}=48)$.

\begin{tabular}{|l|l|c|l|}
\hline \multicolumn{1}{|c|}{ moment } & relationship ISTI $=$ & $\mathrm{r}$ & \multicolumn{1}{c|}{$\mathrm{p}$} \\
\hline before infusion & $-13.5 \cdot \mathrm{CO}+211$ & 0.5453 & $<0.025$ \\
\hline after $250 \mathrm{ml}$ & $-10.1 \cdot \mathrm{CO}+201$ & 0.4920 & $<0.05$ \\
\hline after $500 \mathrm{ml}$ & $-9.5 \cdot \mathrm{CO}+197$ & 0.4609 & $<0.05$ \\
\hline pooled data & $-10.7 \cdot \mathrm{CO}+202$ & 0.4917 & $<0.005$ \\
\hline
\end{tabular}

With respect to changes in ISTI and CO it was found that $\Delta$ ISTI was inversely related to $\Delta \mathrm{CO}$. The relationship between the moments 1 and 3 was found to be significant, both in absolute and relative measure $(\mathrm{r}=0.5304 ; \mathrm{N}=16$; $\mathrm{p}<0.025)$. Expressed as a percentage of change this relationship was: $\Delta \mathrm{ISTI}_{31}=-0.70 \cdot \Delta \mathrm{CO}_{31}+6.8 \%$. The relationship between the changes in both parameters from the moments 1 to 2 was found to be similar however not significant, probably because of lack of power of the study. The effect of fluid administration on all parameters between the moments 2 and 3 was minimal. The cardiac output did hardly change: $\Delta \mathrm{CO}_{32}=0.0 \pm 0.3 \mathrm{l} / \mathrm{min}$.

\section{Discussion}

The Initial Systolic Time Interval (ISTI) can be considered as a measure for the time delay between the electrical and mechanical activity of the heart [6]. Little is known about the clinical relevance of this period. This paper introduces a method using ISTI to evaluate and predict the circulatory response to fluid administration in patients after cardiac surgery and presents preliminary results of a pilot study comparing this parameter with $\mathrm{CO}$ responsiveness. Although the power of the study was low, the preliminary results showed significant relationships between the ISTI and the cardiac output at any moment during the fluid 
administration procedure. Further, a significant relationship between the changes $\Delta \mathrm{ISTI}$ and $\Delta \mathrm{CO}$ before and after full fluid administration was found. This indicates that the ISTI is dependent upon preload, indirectly reflecting cardiac output via the Frank-Starling mechanism, and that ISTI has the potential to be used as a clinical parameter assessing preload. The main response in $\mathrm{CO}$ and ISTI occurred between the moments 1 and 2 indicating that the second administration of fluid between moment 2 and 3 was redundant in most patients.

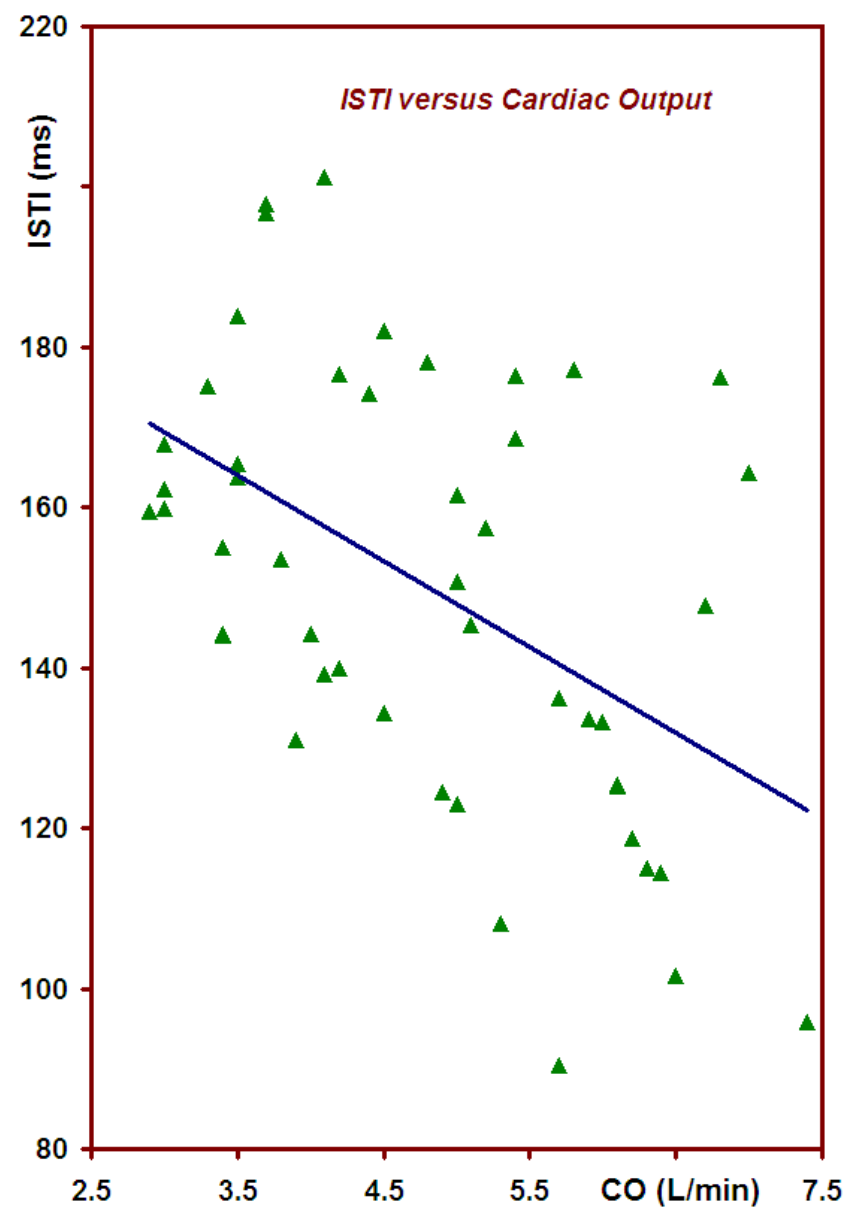

Fig.3: A significant relationship between the Initial Systolic Time Interval (ISTI) and the Cardiac Output (CO) was found before and after intravenous administration of $2 \times 250 \mathrm{ml}$ of a Gelofusine ${ }^{\circledR}$ solution in two steps of $250 \mathrm{ml}$ each.

No significant or systematic relationships between PTT and $\mathrm{CO}$ or $\triangle \mathrm{PTT}$ and $\Delta \mathrm{CO}$ were found. This is in contrast with earlier reports $[8,9]$. Probably, this absence originates from the limited power of this study to detect significances. The number of patients who responded to the fluid administration was small. The mean response in cardiac output to fluid administration was also low and the accuracy of measurement of PTT by means of a catheter in the arteria radialis is limited. The time needed for the systolic pressure wave to reach the catheter tip (VTT) is substantial, varies between individuals and is subject to sympathetic activity [11]. This suggests that measurement of the PTT is of limited value for clinical assessment of preload and of the effect of volume expansion. It is concluded that ISTI is dependent upon preload, and that ISTI has the potential to be used as a parameter assessing preload. Further research comprising a larger number of patients will elucidate its usefulness in clinical practice.

\section{References}

1. Patterson RP. Impedance cardiography: What is the source of the signal? J. Phys.: Conf. Ser. 2010;224:012118 http://dx.doi.org/10.1088/1742-6596/224/1/012118

2. Lababidi Z, Ehmke DA, Durnin RE, Leaverton PE, Lauer RM. The first derivative thoracic impedance cardiogram. Circulation 1970;41:651-8

3. Burgess HJ, Penev PD, Schneider R, Van Cauter E. Estimating cardiac autonomic activity during sleep: impedance cardiography, spectral analysis, and Poincaré plots. Clin Neurophysiol 2004;115:19-28

4. Schweiger E, Wittling W, Genzel S, Block A. Relationship between sympathovagal tone and personality traits. Person Individ Diff 1998;25:327-37

5. Schäginger $\mathrm{H}$, Weinbacher $\mathrm{M}$, Kiss A, Ritz R, Langewitz W. Cardiovascular indices of peripheral and central sympathetic activation. Psychosom Med 2001;63:788-96

6. Meijer JH, Boesveldt S, Elbertse E, Berendse HW. Method to measure autonomic control of cardiac function using time interval parameters from impedance cardiography. Physiol Meas 2008;29: S383-91

7. Reuter DA, Felbinger TW, Schmidt C, Kilger E, Goedje O, Lamm P, Goetz AE. Stroke volume variations for assessment of cardiac responsiveness to volume loading in mechanically ventilated patients after cardiac surgery. Intensive Care Med 2002;28:392-8

8. Bendjelid K, Suter PM, Romand JA. The respiratory change in preejection period: a new method to predict fluid responsiveness. J Appl Physiol 2004;96:337-42

9. Feissel M, Badie J, Merlani PG, Faller JP, Benjelid K. Pre-ejection period variations predict the fluid responsiveness of septic ventilated patients. 2005 Crit Care Med 2005;33:2534-9

10 Meijer JH, Hoekstra F， Habers E， Verdaasdonk RM, Janssen TWJ (2010). Dynamic Response of the Initial Systolic Time Interval to a Breathing Stimulus measured with Impedance Cardiography. J Phys: Conf Ser 2010;224:012119.

http://dx.doi.org/10.1088/1742-6596/224/1/012119

11 Chan GSH, Middleton PM, Celler BG, Wang L, Lovell NH. Change in pulse transit time and pre-ejection period during head-up tilt-induced progressive central hypovolaemia. J Clin Monit Comput 2007;21:283-93

12 Smorenberg A, Lust EJ, Verdaasdonk RM, Groeneveld ABJ, Meijer JH. Prediction and monitoring of fluid responsiveness after coronary bypass surgery using the Initial Systolic Time Interval: Preliminary Results. J Phys: Conf Ser 2010;224:012122. http://dx.doi.org/10.1088/1742-6596/224/1/012122

13 Meijer JH, Reulen JPH, Oe PL, Allon W, Thijs LG, Schneider H. Differential impedance plethysmography for measuring thoracic impedances. Med Biol Eng Comput 1982;20:187-94 